\title{
ARTICLE
}

\section{Display of overlapping mesh tallies in Moritz}

\author{
Kenneth A. Van Riper ${ }^{*}$ \\ White Rock Science, Los Alamos, NM, 87544, USA
}

\begin{abstract}
We describe new features that enhance the display of mesh tallies in the Moritz geometry editing and visualization program. The use of these features is demonstrated in two-dimensional plots of the results of Monte Carlo evaluations of shielding surrounding a proton therapy treatment room. In regions where two or more mesh tallies overlap, a new method plots the value with the lowest relative error. Contour lines may be plotted over the color-coded mesh tally plots. For each contour value, the program chooses a contour color that is distinct from the underlying mesh tally. Interpolation may be used to smooth the mesh tally plot and straighten the contours. A method to extract tally values at specified locations speeds comparisons with analytic shielding calculations. Enhancements for multiple tallies include the ability to read multiple tally files, selecting visibility by particle, and changing the data type plotted for all tallies. Division of two mesh tallies may be used for comparison of runs on different computers or with different code versions. The user can restrict the minimum and maximum of the values that are plotted. The user can set a maximum/minimum ratio beyond which logarithmic scaling is used and can choose whether or not the data is plotted after reading a mesh tally file.
\end{abstract}

Keywords: bremsstrahlung; Monte Carlo; Moritz; mesh tally; shielding

\section{Introduction}

Moritz is a geometry editing and display program used in support of MCNP [1], MCNPX [2], and other Monte Carlo transport codes. The geometry is shown in both two-dimensional (2D) and three-dimensional (3D) representations. Transport results, in the form of mesh tallies, can be shown together with the geometry. This paper describes recent enhancements to the 2D mesh tally plots in which the tally values are mapped to smoothly varying colors.

\section{2D Mesh tally plots}

In some cases, different mesh tallies of the same quantity may overlap in in some portions of a $2 \mathrm{D}$ plot. A recent shielding study we performed is illustrative. Penetration of neutrons and photons through thick concrete walls results from a proton beam impinging on a water target inside a rectangular room.

The initial calculation, shown in Figure 1, focused on penetration of the left wall. Biasing through that barrier (using importance splitting) produced excellent (low relative errors $[R E]$ ) results. Penetration of the adjacent back wall, lacking biasing there, was not well characterized. A calculation of back wall penetration followed. A portion of the mesh tally from the latter calculation, shown in Figure 2, covered the back wall and the back portion of the (now unbiased) left wall.

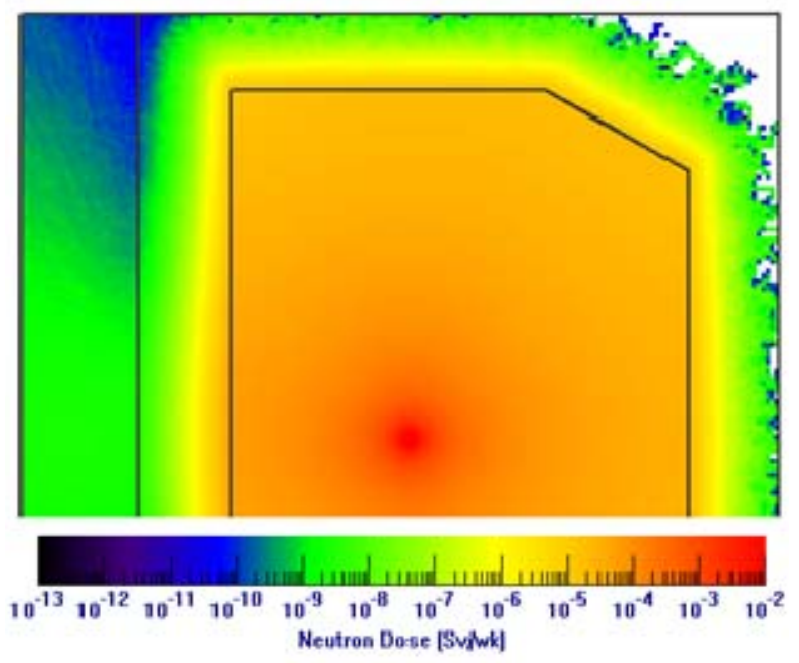

Figure 1. Mesh tally from the calculation of the neutron dose through the left wall. From left to right, the thick black lines separate the hallway, left shielding wall, treatment room, and right wall. The back wall is at the top. 


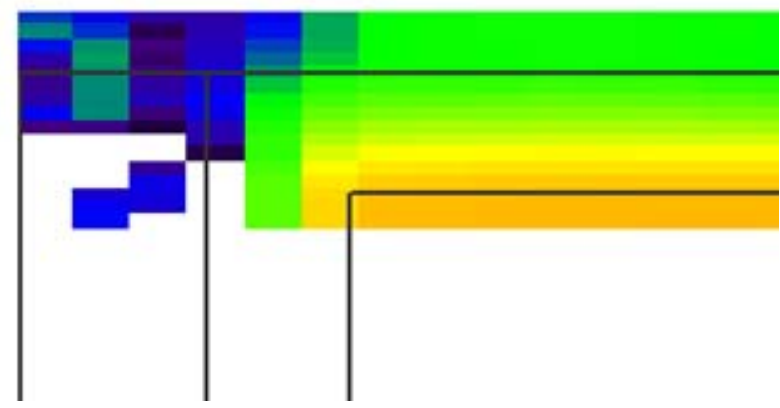

Figure 2. Portion of the mesh tally from the rear wall calculation. The rear wall is bordered by the 2 horizontal black lines.

\subsection{Overlapping mesh tallies}

When plotting 2D mesh tallies, the original Moritz action was to plot one mesh tally over previously plotted tallies. The plotting order followed the order in which the tallies were loaded. In our example, the left and back wall tallies overlap in the left rear corner where portions of each tally have large REs. Figure 3 shows the resulting plot. Because of the large errors, the tally values may show large fluctuations between adjacent mesh cells instead of the smooth variation that is expected in a good simulation.

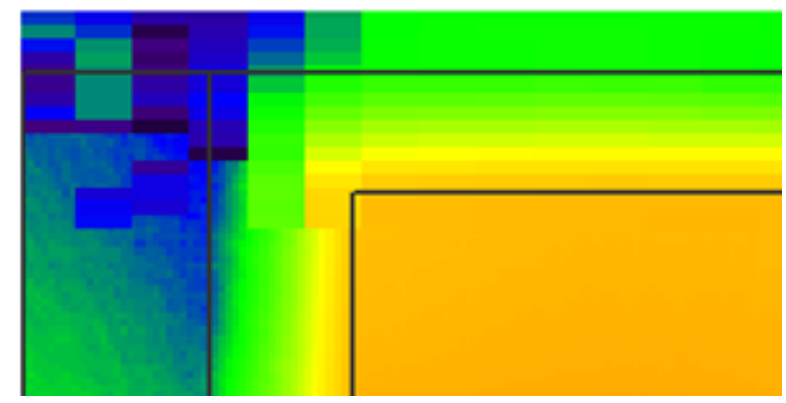

Figure 3. The rear wall mesh tally overlaid on the left wall mesh tally.

\subsection{Merging overlapping mesh tallies}

A new plotting algorithm was developed that plots the tally value with the lowest RE in regions where two or more mesh tallies overlap. If no tallies overlap, the original plotting method is used. Because the mesh cells of different tallies may not exactly coincide, the comparison is performed at each pixel of the $2 \mathrm{D}$ plot. If any of the overlapping tallies has a low RE in the overlap region, the plot will not show large fluctuations. Figure 4 shows the same two mesh tallies as Figure 3 but plotted using the RE merging algorithm. The same $\mathrm{RE}$ comparison method is used when plotting the RE rather than the tally value. The new algorithm plots more slowly than the original but the display time is acceptable (seconds rather than less than a secon

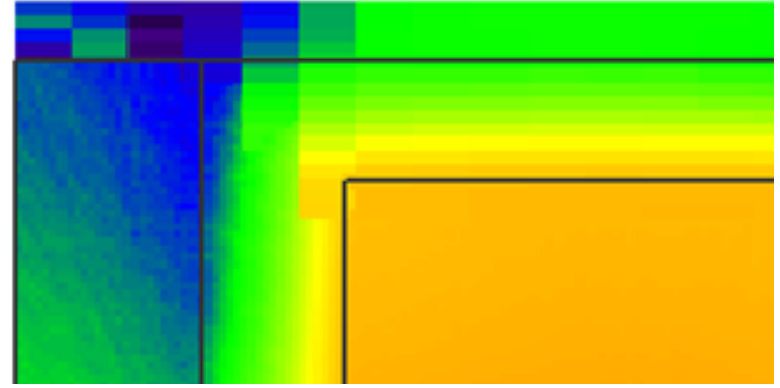

Figure 4 . The rear wall mesh tally merged with the left wall mesh tally.

\section{2D mesh tally contour lines}

Contour lines can be drawn over 2D mesh tally plots. Figure 5 shows an example. The contour values may be shown as vertical lines in the color legend bar below the plot. The contour values can be typed into a dialog or read from a file.

The contour lines can be drawn using a single color or, as in Figure 5, plotted using a method that chooses a color distinct from the underlying mesh tally color. In this method, the color is offset from the color of the contour value by the user specified offset value $o$. The mesh tally values are mapped to colors $C(i)$ where $0 \leq i$ $\leq 255$. A contour value corresponds to the color $\mathrm{C}(\mathrm{j})$. The contour color is $C(k)$ where $k=(j+o) \% 256$ and \% is the modulus operator. This method ensures that the contour color is different than the underlying mesh tally color. The recommended value is $o=128$.

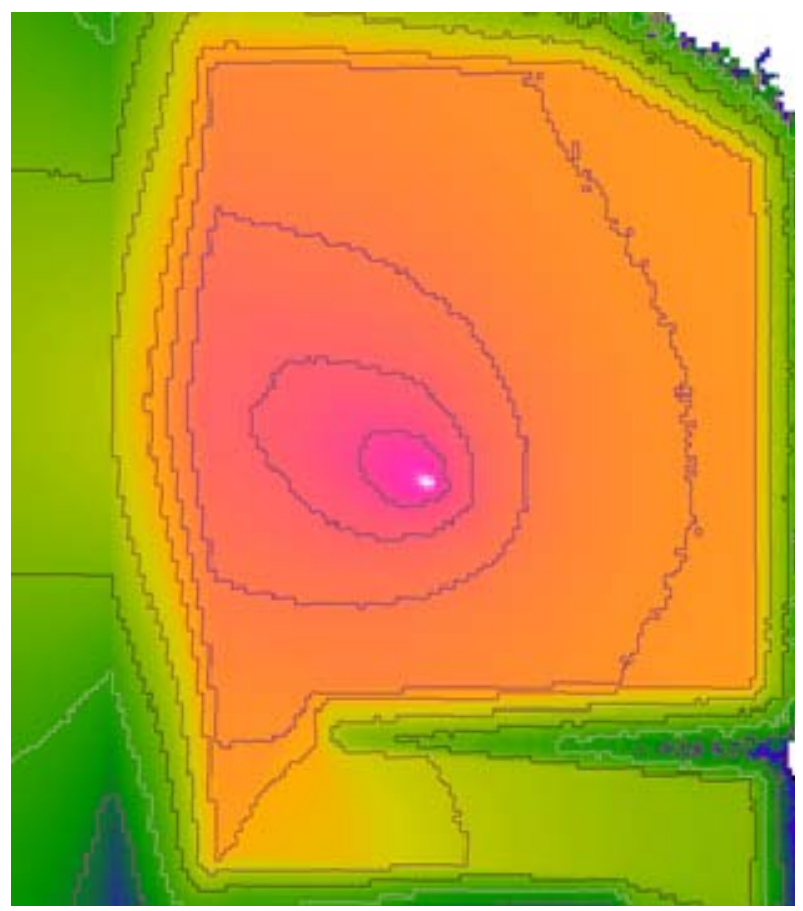

Figure 5. Mesh tally plot with contour lines.

Future work includes adding labels along the lines to show the value, adding contours in $3 \mathrm{D}$ and to data 
planes, and a contour legend window.

\section{2D mesh tally interpolation}

The 2D mesh tally plots can use interpolation to lessen the sharp jump in color from one mesh cell to another and to smooth contour lines. The interpolation scheme averages the value in a mesh cell with the values in its 26 nearest neighbors (skipping neighbors that are outside of the mesh). The values are weighted by the distance from the mesh cell center to the neighbors' centers. If logarithmic scaling is in effect, the logarithm of the value is interpolated and no interpolation is done if the value is nonpositive. The same interpolation method is used when plotting the relative error.

Figure 6 shows a mesh tally and contour lines with interpolation. The finite size of the mesh cells is still apparent but the effect is lessened with interpolation. The contours are much smoother with interpolation.

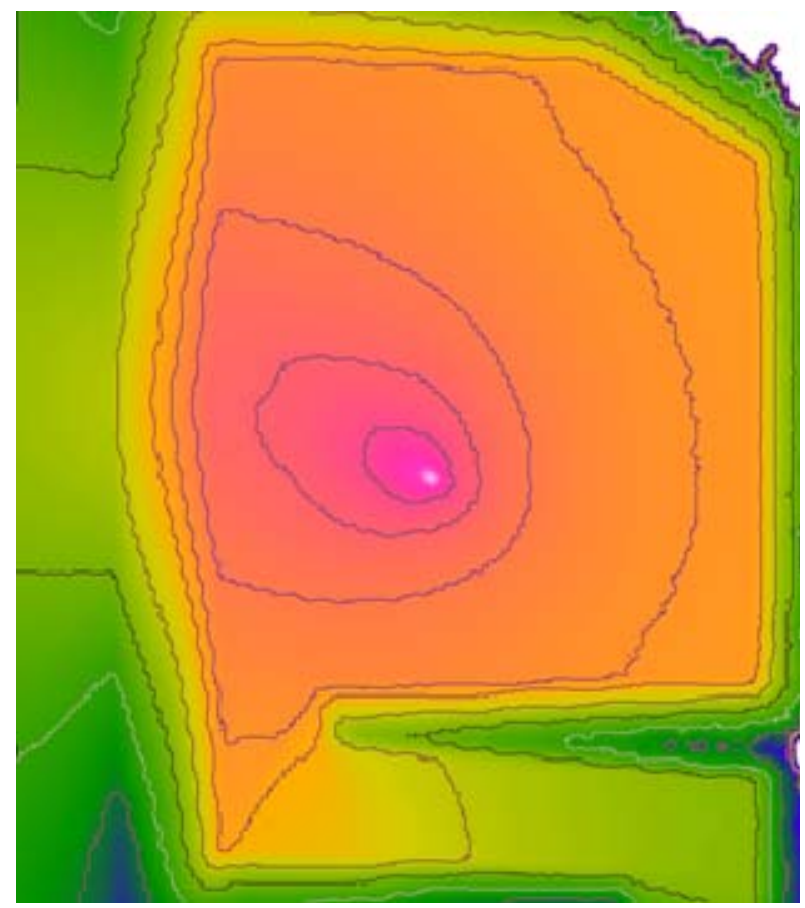

Figure 6. Mesh tally plot with contour lines using interpolation.

\section{Other mesh tally enhancements}

\subsection{Tally values at specified coordinates}

In addition to the Monte Carlo Shielding analysis, other team members evaluated the shielding using analytic methods. A comparison of the two methods requires the dose values from the Monte Carlo calculations at specific locations. Moritz shows the value of the mesh tally at the position of the cursor when it is in a $2 \mathrm{D}$ window. For a large number of locations, accurately positioning the cursor and copying the values is a tedious and time-consuming process. In order to obtain the results much more quickly, we added the ability to enter one or more locations and obtain the mesh tally values (and REs) from one or all mesh tallies. Multiple coordinates may be read from a file or a single location may be typed into a dialog. The output is written to a file and/or to a program window.

\subsection{Multiple tallies from multiple files}

The shielding project required several additional calculations after the left wall and rear wall studies described above. Using and keeping track of a large number of mesh tallies for different particles required some new features in Moritz. When reading a second mesh tally file, the original action taken by Moritz was to replace any existing mesh tally data with the new data. We added the ability to retain existing data, changing mesh numbers if necessary.

When selecting a tally for plotting, the program shows both the mesh number and particle name for a tally. To keep track of new tallies defined by the addition of two others, "SumN" replaces the particle name. $\mathrm{N}$ is the number of tallies that have been added together. For example, adding a neutron tally and a photon tally results in a Sum2 tally; adding that to a proton tally gives a Sum3 tally.

In addition to setting the visibility and whether to plot the data or its RE for each tally, we added operations that affect multiple tallies. Only those tallies for a selected particle (including the Sums) can be shown; those for other particles are hidden. All tallies can be hidden in a single operation. The data or RE choice can be made for all tallies.

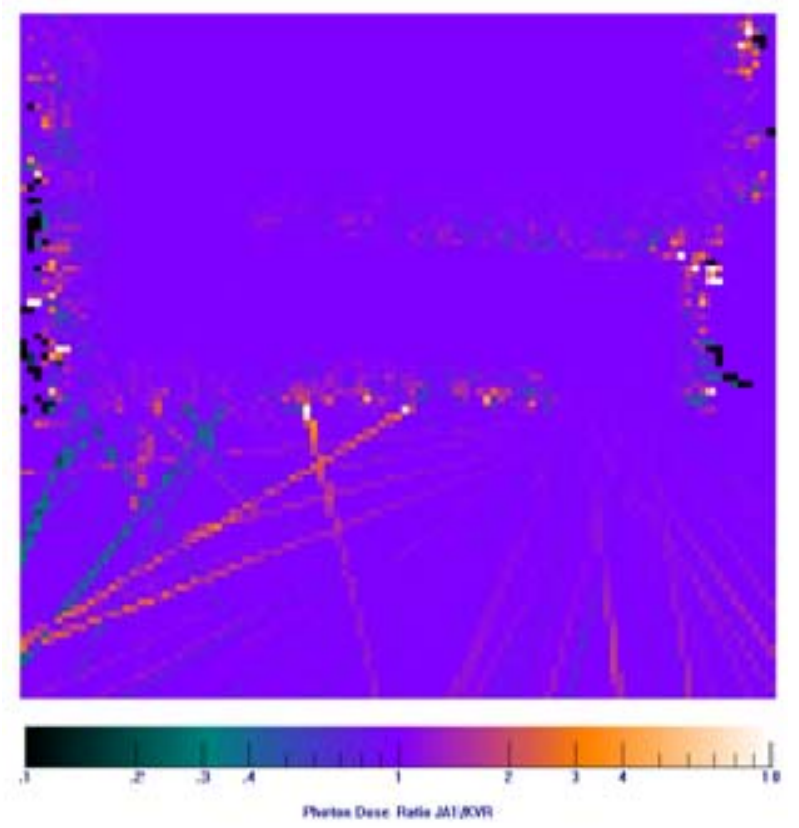

Figure 7. Ratio of photon dose mesh tallies calculated on 2 different computers.

\subsection{Mesh tally comparison}

We extended the creation of a new mesh tally from 
the combination of two others to use subtraction, multiplication, and division as well as the addition operator. Division is useful for comparing mesh tallies from runs using different code versions or the same problem run on different computers.

Figure 7 shows the division of two runs made on different computers. The tallies agree (i.e. quotient $=1$ ) where the statistics are good. Because these calculations used a relatively low number of histories, the REs in and beyond the shielding walls are large and the comparison is not exact in those regions.

\subsection{New display options}

Moritz had the option of not plotting values less than or equal to zero when logarithmic scaling is used. The choice affects both the 2D and 3D plots. A number of users have requested additional flexibility. We have extended the $\leq 0$ choice to linear scaling. Values less than the plot minimum or greater than the maximum can be hidden, as well as values less than or greater than user specified values. Tallies can be plotted or not immediately after they are loaded. These options can be set separately for the 2D and 3D plots.

The user can set a maximum/minimum ratio beyond which logarithmic scaling is used (the minimum does not include zero values).

\section{Conclusion}

The new mesh tally features we added to Moritz enhanced the quality of plots prepared for the sponsor of the shielding project and permitted them to be prepared more quickly. These features will be very helpful to the author and other users in future shielding projects.

\section{Acknowledgements}

The authors wish to thank Jake Anderson for the use of his SciAAS High Performance Compute Cluster.

The Moritz program is available from White Rock Science - www.whiterockscience.com

\section{References}

[1] X-5 Monte Carlo Team, MCNP - A General Monte Carlo N-Particle Transport Code, Version 5 Volume I: Overview and Theory, LA-UR-03-1987, Los Alamos National Laboratory, revised 2/2008 (2008).

[2] D. Pelowitz (ed.), MCNPX User's Manual, Version 2.7.0, Los Alamos National Laboratory, LA-CP-11-00438 (2011). 